\title{
Phase Diagram of Superconductivity on the Anisotropic Triangular Lattice Hubbard Model
}

\author{
Hiori KINO and Hiroshi KonTANI ${ }^{1}$ \\ Joint Research Center for Atom Technology, Tsukuba, Ibaraki, 305-8562, Japan. \\ ${ }^{1}$ Institute for Solid State Physics, University of Tokyo, 7-22-1 Roppongi, Minato-ku, Tokyo 106-8666, Japan.
}

(September 11, 2018)

We study the electronic states of the anisotropic triangular lattice Hubbard model at half filling, which is a simple effective model for the organic superconducting $\kappa$-BEDT-TTF compounds. We treat the effect of the Coulomb interaction by the fluctuation exchange (FLEX) method, and obtain the phase diagram of this model for various sets of parameters. It is shown that the $d$-wave superconductivity is realized in the wide region of the phase diagram, next to the antiferromagnetic states. The obtained phase diagram explains the characters of the experimental results very well.

PACS numbers: 74.70.Kn, 74.20.-z, 74.25.Dw, 75.50.Ee

It is known that the layered organic compounds made of BEDT-TTF molecules exhibit rich variety of ground states, through the strong correlation effects between electrons. Among them, $\kappa$-polytype of BEDT-TTF systems has attracted much attention because many of them are superconducting materials. The superconducting transition temperature $\left(T_{c}\right)$ reaches $\sim 10 \mathrm{~K}$, whose ratio to the band width is as large as that of the high- $T_{\mathrm{c}}$ cuprates.

In fig. 1, we show the schematic pressure-temperature phase diagram of the $\kappa$-(BEDT-TTF) compounds. [1] For example, $\mathrm{Cu}\left[\mathrm{N}(\mathrm{CN})_{2}\right] \mathrm{Cl}$ salt at ambient pressure is in the antiferromagnetic (AF) insulating phase as shown by the arrow in the figure. The Néel temperature $\left(T_{\mathrm{N}}\right)$ is $27 \mathrm{~K}$ and the spin moment is greater than $0.4 \mu_{\mathrm{B}}$ per magnetic unit, i.e., dimer of BEDT-TTF molecules. The antiferromagnetism disappears with increasing pressure to give birth to the superconductivity. $T_{\mathrm{c}}$ takes its maximum value, $13 \mathrm{~K}$, at $200 \mathrm{bar}$, but this superconducting (SC) phase also suppressed by several kbar, where a paramagnetic metallic phase is stabilized. This means that the superconductivity coexists with the strong antiferromagnetic correlations. Thus, it is natural to consider that the antiferromagnetic fluctuation is the origin of superconductivity.

In the previous studies, the organic compound has been studied theoretically through the local density approximation and the Hartree-Fock approximation. [2, 3] The latter study could explain the pressure dependence of the AF boundary in fig. 11. The structures of these compounds are very complex. Nonetheless, the electronic structure near the Fermi level is very simple, because it is composed of well separated HOMO levels. The simplest effective model is a triangular lattice Hubbard model with anisotropic hoppings at half filling, as shown below. The effective on-site Coulomb repulsion $(U)$ is estimated to be comparable with the band width $(W)$ in this model.

The aim of this letter is to discuss the mechanism of the superconductivity, and to understand the entire phase diagram shown in fig. 1. For this purpose, we study the anisotropic triangular lattice Hubbard model by using the fluctuation exchange (FLEX) method. It is one of the methods that have much advantage for the systems with large spin fluctuations and that can handle the SC and $\mathrm{AF}$ states on the same footing. In this letter, we find the $d$-wave $\mathrm{SC}$ phase next to the $\mathrm{AF}$ phase. The obtained $T_{c}$ is consistent with experiments. This is the first systematic study of the organic SC compounds by using the FLEX method.

The FLEX method is a kind of self-consistent perturbation theory with respect to $U$, which gives reliable results although it is an approximation. For example, the imaginary time Green function obtained by the FLEX method agrees well with that by the QMC for the square lattice Hubbard model with moderate $U$. [4] This method has been applied to the study of high- $T_{\mathrm{c}}$, and various non-Fermi liquid behaviors are reproduced well. 5 11 Recently it has also been applied to the superconducting ladder compound, $\mathrm{Sr}_{14-x} \mathrm{Ca}_{x} \mathrm{Cu}_{24} \mathrm{O}_{41}$. [12]

Dimerization of $\kappa$-(BEDT-TTF) salts is so large that we can take a dimer of BEDT-TTF molecules as a unit. So, as a simple effective model, we consider the twodimensional single band Hubbard model with the lattice structure shown in fig. 2. where $t_{0}, t_{1}, t_{2}$ are hopping parameters. We also take into account the on-site Coulomb repulsion $(U)$, which is an energy loss when two electrons are on the same BEDT-TTF dimer in the original $\kappa$-(BEDT-TTF) lattice. The hopping parameters depend on materials, but both the band calculations and experiments indicate that $t_{1} \sim t_{0}$ and $\left|t_{2}\right| \ll\left|t_{0}\right|,\left|t_{1}\right|$. [2 13 13 In this study, we put $t_{0}=-1, t_{2}=-0.05$, and change $t_{1}(\mathrm{i} 0)$ as a parameter. In this sense, this model is an anisotropic triangular lattice. For $\left|t_{1}\right| \leq\left|t_{0}\right|, W$ is always $8\left|t_{0}\right|$. A similar model has been studied recently, but the essence of the physics of $\kappa$-(BEDT-TTF) salts is contained in the above simple model. [16]

Here, we explain the FLEX method. The Dyson equation is written as

$$
\left\{G\left(\mathbf{k}, \epsilon_{n}\right)\right\}^{-1}=\left\{G^{0}\left(\mathbf{k}, \epsilon_{n}\right)\right\}^{-1}-\Sigma\left(\mathbf{k}, \epsilon_{n}\right),
$$

where $G^{0}(\mathbf{k}, \epsilon)$ is the unperturbed Green function. The self-energy is given by 


$$
\begin{aligned}
& \Sigma\left(\mathbf{k}, \epsilon_{n}\right)=T \sum_{\mathbf{q}, l} G\left(\mathbf{k}-\mathbf{q}, \epsilon_{n}-\omega_{l}\right) \cdot U^{2} \\
& \times\left(\frac{3}{2} \chi^{(-)}\left(\mathbf{q}, \omega_{l}\right)+\frac{1}{2} \chi^{(+)}\left(\mathbf{q}, \omega_{l}\right)-\chi^{0}\left(\mathbf{q}, \omega_{l}\right)\right), \\
& \chi^{( \pm)}\left(\mathbf{q}, \omega_{l}\right)=\chi^{0} \cdot\left\{\hat{1} \pm U \chi^{0}\left(\mathbf{q}, \omega_{l}\right)\right\}^{-1}, \\
& \chi^{0}\left(\mathbf{q}, \omega_{l}\right)=-T \sum_{\mathbf{k}, n} G\left(\mathbf{q}+\mathbf{k}, \omega_{l}+\epsilon_{n}\right) G\left(\mathbf{k}, \epsilon_{n}\right),
\end{aligned}
$$

where $\epsilon_{n}=(2 n+1) \pi T$ and $\omega_{l}=2 l \pi T$, respectively. We solve the equations (1)-(4) self-consistently, choosing the chemical potential $\mu$ so as to keep the system at halffilling.

To determine $T_{N}$, we calculate the Stoner factor without vertex corrections, $\alpha_{\mathrm{S}}$, given by

$$
\alpha_{\mathrm{S}}=\max _{\mathbf{k}}\left\{U \cdot \chi^{0}(\mathbf{k}, \omega=0)\right\},
$$

The antiferromagnetic critical points are determined by the Stoner criterion, $\alpha_{\mathrm{S}}=1$.

We also determine $T_{\mathrm{c}}$ by solving the linearized Eliashberg equation with respect to the singlet-pairing order parameter, $\phi\left(-\mathbf{k}, \epsilon_{n}\right)=+\phi\left(\mathbf{k}, \epsilon_{n}\right)$,

$$
\begin{aligned}
& \lambda \cdot \phi\left(\mathbf{k}, \epsilon_{n}\right)=-T \sum_{\mathbf{q}, m} V\left(\mathbf{k}-\mathbf{q}, \epsilon_{n}-\epsilon_{m}\right) \\
& \quad \times G\left(\mathbf{q}, \epsilon_{m}\right) G\left(-\mathbf{q},-\epsilon_{m}\right) \cdot \phi\left(\mathbf{q}, \epsilon_{m}\right), \\
& V\left(\mathbf{k}, \omega_{l}\right)=\frac{3}{2} U^{2} \chi^{(-)}\left(\mathbf{k}, \omega_{l}\right)-\frac{1}{2} U^{2} \chi^{(+)}\left(\mathbf{k}, \omega_{l}\right)+U,
\end{aligned}
$$

where $T_{\mathrm{c}}$ is given by the condition that $\lambda=1$.

The theories by Mermin, Wagner and Hohenberg prohibits finite $T_{\mathrm{N}}$ and $T_{\mathrm{c}}$ in two dimension. [17, 18] It is well known that $\alpha_{\mathrm{S}}$ given by eq. (5) satisfies this condition, because FLEX treats the spin fluctuations self-consistently. [7.9 So we determine $T_{\mathrm{N}}$ by the condition $\alpha_{\mathrm{S}}=\alpha_{\mathrm{N}}$, where we set $\alpha_{\mathrm{N}}$ as $\left(1-\alpha_{\mathrm{N}}\right)^{-1} \sim O(100)$. The AF state will occur through the weak coupling between layers. On the other hand, $\lambda=1$ is fulfilled at finite $T_{\mathrm{c}}$ by using eq.(6). The obtained $T_{\mathrm{c}}$ is, however, reliable in many cases.

First, let us see the phase diagram as a function of $\left|t_{1}\right|\left(=-t_{1}\right), U$ and $T$, for $T \geq 0.02$. A number of sections of the phase diagram are plotted in fig.3. $16 \times 16$ $k$-points and 256 Matsubara frequencies (abbreviated as $16 \times 16 \times 128 \mathrm{mesh}$ ) are used to draw this phase diagram. For $32 \times 32 \times 1024$ or $64 \times 64 \times 512$ mesh the Stoner factor becomes larger, but that effect can be compensated by taking slightly larger $\alpha_{N}$. In fig. 3 we set $\alpha_{N}=0.99$. As for the superconductivity, the size effect of $T_{\mathrm{c}}$ is rather smaller than that of $T_{\mathrm{N}}$, because $\lambda$ rapidly grows near the critical temperature.

Fig. 3(a) shows the critical temperatures, $T_{\mathrm{N}}$ (small circles) and $T_{\mathrm{c}}$ (large circles), on the planes of $U$ vs $T$ at several values of $\left|t_{1}\right|\left(=-t_{1}\right)$. Only AF phase appears at $\left|t_{1}\right|=0.3$, SC occurs for $\left|t_{1}\right|=0.4 \sim 0.7$ on the left hand side of the AF phases. As $\left|t_{1}\right|$ increases, we see the followings: The onset value of $U$ for the AF region shifts larger, at the same time, the peak of $T_{\mathrm{N}}$ decreases, and the SC region are extended. The maximum value of $T_{\mathrm{c}}$ at each section increases for $\left|t_{1}\right|<0.6$, and then decreases for $\left|t_{1}\right|>0.6$.

Fig.3(b) shows $T_{\mathrm{c}}$ and $T_{\mathrm{N}}$ on the planes of $\left|t_{1}\right|$ vs $T$, at $U=3.0,6.0$ and 9.0 for $T \geq 0.02$. At $U=3.0$ only the AF phase appears. On the other hand, the SC state occurs next to the AF phases at $U=6.0$ and 9.0. As $U$ increases, both the SC and AF boundaries move toward larger $\left|t_{1}\right|$ region. At $U=9.0$, which is the largest $U$ in this figure, $T_{\mathrm{N}}$ decreases as $\left|t_{1}\right|$ becomes larger and the SC state takes place instead of the AF state for $\left|t_{1}\right|>0.7$. This SC phase, however, disappears for $\left|t_{1}\right|>0.75$. At the $\left|t_{1}\right|=0.8$, the $\mathrm{AF}$ occurs at larger $U$, but no SC state is stabilized at $T \geq 0.02$. No SC and AF phases appear at $\left|t_{1}\right|=0.9$ and 1.0 for $U \leq 40$. We will discuss the reason for this in the latter section. The value of $\lambda$ increases monotonically not only as $T$ is decreased. but also as decreasing $\left|t_{1}\right|$ as shown in fig. 3

Next in fig. 1 , we plot the SC order parameter, $\phi(k, \omega=$ 0 ), along the line with the arrows on the Fermi surface. The parameters used in fig. (1) are $U=4.6, t_{1}=-0.5$ and $T=0.024$, where $\lambda=1.02$. One can clearly see that there are nodes in the $(\pi, \pi)$ and $(-\pi, \pi)$ directions and the superconductivity has $d_{x^{2}-y^{2}}$-like symmetry. The maximum values of $\phi\left(k_{F}, \omega=0\right)$ is slightly shifted from $(0, \pi)$ direction to $(-\pi, \pi)$ direction. This is also the case for all the other superconducting phases. We have checked that the odd-parity superconductivity is not realized in the present model.

To understand the overall aspects of the phase diagram, we discuss it on the basis of the weak coupling theory. In this scheme, the effective interaction between a singlet Cooper pair is given by

$$
\lambda_{\mathrm{w}}=-\max _{\phi}\left\langle V\left(q-q^{\prime}, 0\right) \phi(q) \phi\left(q^{\prime}\right)\right\rangle_{\mathrm{FS}} /\left\langle|\phi(q)|^{2}\right\rangle_{\mathrm{FS}},
$$

where $\phi(q)$ is the energy-independent SC order parameter, and $V(q, \omega)$ is given by eq.(7). The obtained $\phi(q)$ has the d-wave-like symmetry in this model as shown above. Introducing the cutoff energy $\omega_{c}$, the $T_{c}$ within the weak coupling theory is given by $T_{c}=1.14 \omega_{c} \exp \left(-1 / \lambda_{\mathrm{w}}\right)$.

In fig. 5 , we show the obtained $\chi^{(-)}(q, \omega=0)$ by the FLEX method for $\left|t_{1}\right|=0.3 \sim 0.7$. At each $\left|t_{1}\right|$, we choose $U$ so as to satisfy $\alpha_{S}=0.99$. Then, $V(q) \approx$ $3 U^{2} / 2 \cdot \chi^{(-)}(q, 0)$ and $\max _{q}\{V(q)\} \approx 3 U / 2\left(1-\alpha_{S}\right)$. For each $\left|t_{1}\right|, \chi^{(-)}(q, 0)$ shows a sharp peak around $(\pi, \pi)$, which favors the $d$-wave SC state similarly to the high- $T_{\mathrm{c}}$ cuprates. We note that $\chi^{(-)}(q, 0)$ has an incommensurate structure when $\alpha_{S}$ is rather small.

As $\left|t_{1}\right|$ increases from 0.3 to 0.7 , the peak of $\chi^{(-)}(q, 0)$ around $(\pi, \pi)$ becomes broader, because the nesting condition becomes worse. This situation will make $T_{\mathrm{c}}$ lower within the weak coupling scheme. On the other hand, as $\left|t_{1}\right|$ increases, the value of $\max _{q}\{V(q)\}$ becomes larger, which will make $T_{\mathrm{c}}$ higher. The optimum $\left|t_{1}\right|$ for SC, which is $\left|t_{1}\right|=0.6$ in our calculation, may be determined 
by the balance of these two opposite effects. We have already shown that $d$-wave SC occurs at $T \geq 0.02$ in the case of $\left|t_{1}\right|=0.4 \sim 0.7$. We also find $T_{\mathrm{c}} \approx 0.01$ at $\left|t_{1}\right|=0.8$.

At $\left|t_{1}\right|=0.3$, AF phase completely prevails over the SC phase because $\max _{q}\{V(q)\}$ is rather small at $\alpha_{S}=0.99$. On the other hand, when $\left|t_{1}\right| \gtrsim 0.9, \alpha_{S}=0.99$ is not satisfied for $U<40$. So, $\max _{q}\{V(q)\}$ is also small. Besides, the structure of $V(q)$ changes drastically as shown in the inset of fig.5.: The peak position of $\chi^{(-)}(q, 0)$ shifts from $(\pi, \pi)$ towards $(2 \pi / 3,2 \pi / 3)$, so the AF correlation is violated. If we map our model to the corresponding Heisenberg spin system on the anisotropic triangular lattice, the ground state classically changes from $\mathrm{AF}$ order to the 120 degree structure at $J_{1} / J_{0}=\left(t_{1} / t_{0}\right)^{2}=2 / 3$, i.e., $t_{1} / t_{0}=0.82$. The momentum $(2 \pi / 3,2 \pi / 3)$ corresponds to the 120 degree structure. As a result, for $\left|t_{1}\right| \gtrsim 0.9$, any singlet SC state is not realized up to $U \leq 40$.

The Fermi surfaces at $t_{1}=-0.5$ and $T=0.024$ are drawn in fig.2. The change of the Fermi surface is attributed to the renormalization of $\left|t_{1}\right|$ by many body effect. The sum rule, which must be satisfied in principle, but not necessarily fulfilled in actual numerical calculations, is checked to be satisfied within $0.5 \%$.

Let us discuss the connection between the real materials and the obtained phase diagram. The $T_{\mathrm{c}}$ of $\kappa$-(BEDT$\mathrm{TTF}$ ) is as high as $10 \mathrm{~K}$. If one takes the noninteracting band width $(W)$ to be $5000 \mathrm{~K}$, then $0.03\left|t_{0}\right|=18 \mathrm{~K}$, which corresponds well with the experiments. [1]

Next we consider the effect of pressure. First we return to the original $\kappa$-BEDT-TTF crystal. It is natural to assume that the effect of pressure is most sensitive to the loosely-packed parts, i.e., to the interdimer ones. The interdimer transfer integrals determine the band structure. So $t$ 's or $W$ in the present model is sensitive to the external pressure, while the interdimer transfer integrals and the on-site Coulomb energy on the BEDT-TTF molecule, or $U$ in the effective model, is insensitive to the applied pressure. The ratio of $t_{1} / t_{0}$ will change, but both $t_{0}$ and $t_{1}$ are estimated from the interdmer transfer integrals, thus the ratio does not change so large as $U / W$. Because $W$ is fixed in fig.3, one can regard the pressure effect as decreasing $U$.

Some band calculations suggest that $\left|t_{1}\right| \sim\left|t_{0}\right|$, where no SC and AF phases appear in fig.3, however. 13] One possibility for this discrepancy is that the band calculations estimate the value of $t_{1} / t_{0}$ larger. The other is that the current model is too simple to reflect multiband effects properly. Such a effect is probably taken into account by the renormalization of $t_{1} / t_{0}$ of this effective model, because the $\mathrm{AF}$ phase will emerges at $J_{1} / J_{0} \lesssim 2 / 3$, or $t_{1} / t_{0} \lesssim 0.82$ in the present model as noted above. If one choose such a $t_{1} / t_{0}$, then one can find not only the AF state, but also the $\mathrm{SC}$ state next to the AF phase. Moreover the SC phase appears in higher pressure region. In this sense, the phase diagram in fig. 3 agrees well with the experimental phase diagram.

Furthermore, the experimental AF state is commensu- rate, 1 which is also consistent with the FLEX results that the peak of $\chi(q, 0)$ is always at $(\pi, \pi)$ in the $\mathrm{AF}$ phase, due to the self-consistency. Although the system is half-filling, the region where SC state appears in fig. 3 will not be replaced by the nonmagnetic Mott insulating one, because $U$ is smaller than $W$ in that region. We finally make a comment for chemistries that $T_{\mathrm{c}}$ takes the maximum value, $\sim 0.029$, at $t_{1} \sim 0.6$. $T_{\mathrm{c}}$ possibly becomes higher than that of the currently known materials if one can compose such compounds, though the correspondency between the present simple model and the original complex structure is not straightforward.

In conclusion, we have studied the anisotropic triangular lattice Hubbard models at half-filling by using the FLEX method. We obtained the phase diagram, where the $d$-wave superconducting phase is realized next to the antiferromagnetic one. The resultant phase diagram is consistent with the experimental phase diagram in $\kappa$ (BEDT-TTF) systems. These results suggest that the electron-electron correlation is important in $\kappa$-(BEDTTTF) compounds and that the spin fluctuation mechanism is the possible origin of the superconductivity.

We are grateful to K. Terakura, K. Yamada, K. Ueda, K. Kanoda, K. Yonemitsu, H. Kohno, T. Komatsu and M. Mori for valuable comments and useful discussions and encouragements. This work is partly supported by NEDO.

[1] A recent compact review is K. Kanoda, Physica C 282287 299, (1997).

[2] Y. -N Xu, W. Y. Ching, Y. C. Jean and Y. Lou, Phys. Rev. B 52 12946, (1995).

[3] H. Kino and H. Fukuyama, J. Phys. Soc. Jpn. 652158 , (1996).

[4] N. E. Bickers, D. J. Scalapino and S. R. White, Phys. Rev. Lett. 62 961, (1989).

[5] P. Monthoux and D. Pines, Phys. Rev. B 47 6069, (1993).

[6] P. Monthoux and D. J. Scalapino, Phys. Rev. Lett. 72 1874, (1994).

[7] T. Dahm and L. Tewordt, Phys. Rev. B 52 1297, (1995).

[8] M. Langer, J. Schmalian, S. Grabowski, and K. H. Bennemann, Phys. Rev. Lett. 75 4508, (1995).

[9] J. J. Deisz, D. W. Hess and J. W. Serene, Phys. Rev. Lett. 76 1312, (1996).

[10] S. Koikegami, S. Fujimoto and K. Yamada, J. Phys. Soc. Jpn. 66 1438, (1997).

[11] T. Takimoto and T. Moriya, J. Phys. Soc. Jpn. 66 2459, (1997).

[12] H. Kontani and K. Ueda, Phys. Rev. Lett. 805619 (1998).

[13] e.g., T. Komatsu, N. Matsukawa, T. Inoue and G. Saito, J. Phys. Soc. Jpn. 65 1340, (1996). The overlap integrals are also listed for a number of $\kappa$-(BEDT-TTF) salts and related materials. 
[14] K. Oshima, T. Mori, H. Inokuchi, H. Urayama, H. Yamochi and G. Saito, Phys. Rev. B 38 938, (1988).

[15] Y. Yamauchi, M. V. Kartsovnik, T. Ishiguro, M. Kubota and G. Saito, J. Phys. Soc. Jpn. 65 354, (1996).

[16] In finishing this letter, we noticed the preprint: J. Schmalian, cond-mat/9807042.

[17] N. D. Mermin and H. Wagner, Phys. Rev. Lett. 17 1133, (1966).

[18] P. C. Hohenberg, Phys. Rev. 158 383, (1967).

FIG. 1. Schematic phase diagram of $\kappa$-(BEDT-TTF) compounds.

FIG. 2. A model and the Fermi surface when $t_{1}=-0.5$ at $T=0.024$. The solid line shows the one at $U=4.6$ and the dotted line, $U=0$. Arrows on the solid line are explained in fig.4. $64 \times 64 k$-points and 512 Matsubara frequencies are used.

FIG. 3. Calculated phase diagram on the planes of $U$ vs $T$ at several values of $\left|t_{1}\right|$ (a), and on the planes of $\left|t_{1}\right|$ and $T$ at a few $U$ (b), respectively. The antiferromagnetism is realized in the temperature region below the small circles. and the superconductivity, below the large circles. Lines are guides for the eye.

FIG. 4. The momentum dependence of $\phi\left(k_{F}, \omega=0\right)$ along the line with the arrows on the Fermi surface in fig.2

FIG. 5. $\quad \chi^{(-)}(q, \omega)$ from $q=(0,0)$ to $(\pi, \pi)$ at $t_{1}=-0.3$, $T=0.03$ and $U=2.85$ (solid line), $t_{1}=-0.5, T=0.03$ and $U=5.00$ (broken line) and $t_{1}=-0.7, T=0.03$ and $U=9.60$ (dotted line). $\max _{k} U \chi^{0}(k, \omega=0)$ is around 0.992 at $k=(\pi, \pi)$ for all the cases. The inset shows $\chi^{(-)}(q, \omega)$ from $q=(0,0)$ to $(\pi, \pi)$ at $t_{1}=-1.0, t_{2}=0, T=0.025$ and $U=15$ (solid line) and at $t_{1}=-1.0, t_{2}=0.05, T=0.02$ and $U=15$ (broken line). $\max _{q} U \chi^{0}(q, \omega=0)$ at $t_{1}=-1.0$ is about 0.953 at $q=(2 \pi / 3,2 \pi / 3)$. The $64 \times 64 \times 512$ meshes are used. 


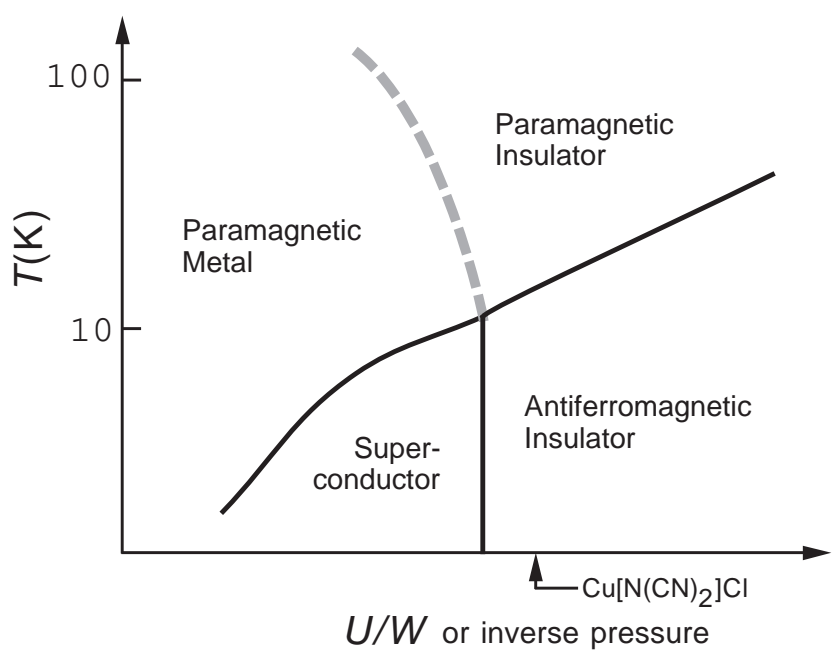

Fig.1

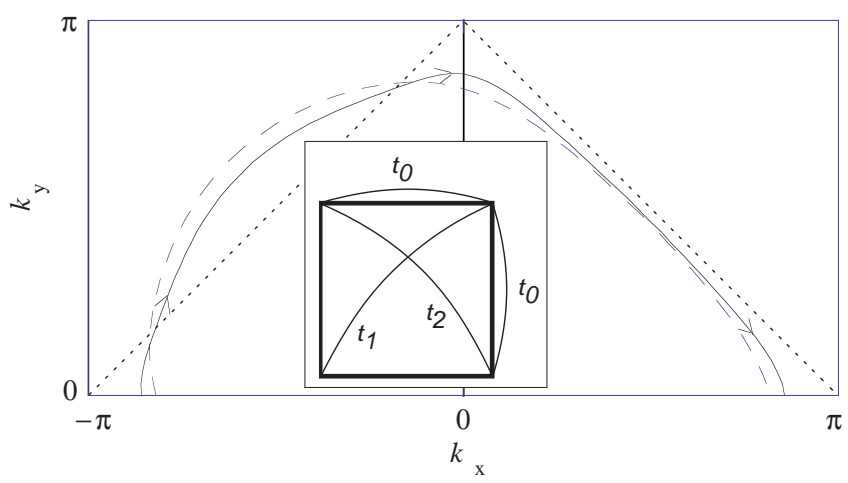

Fig.2

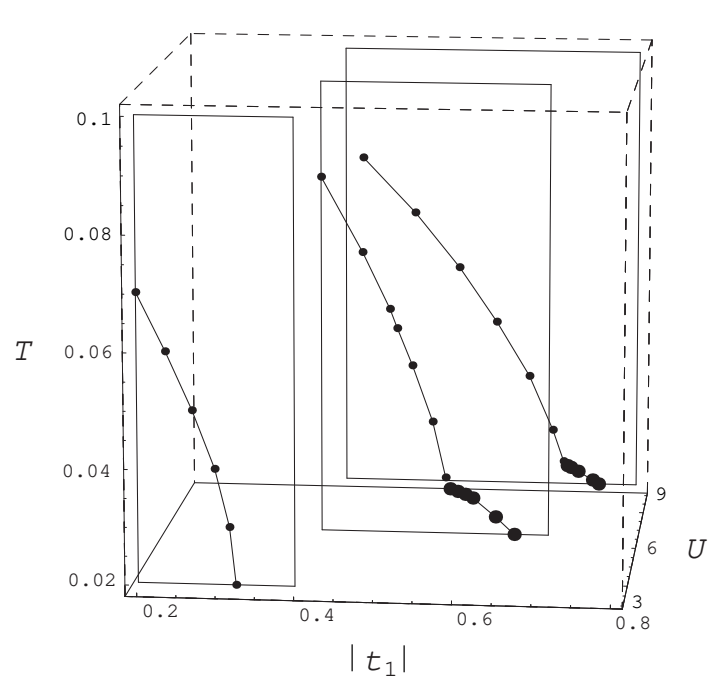

Fig. $3(b)$

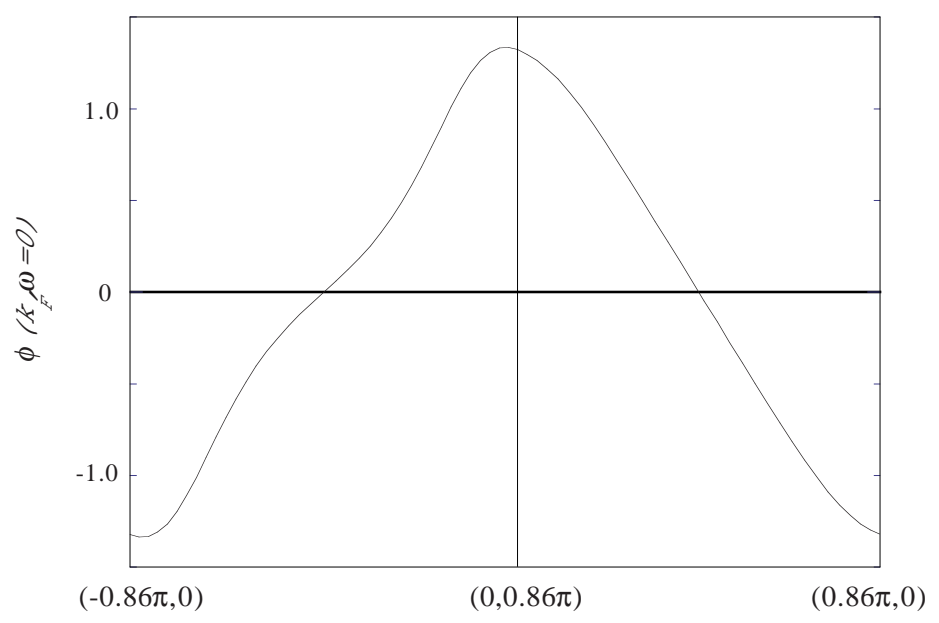

Fig. 4

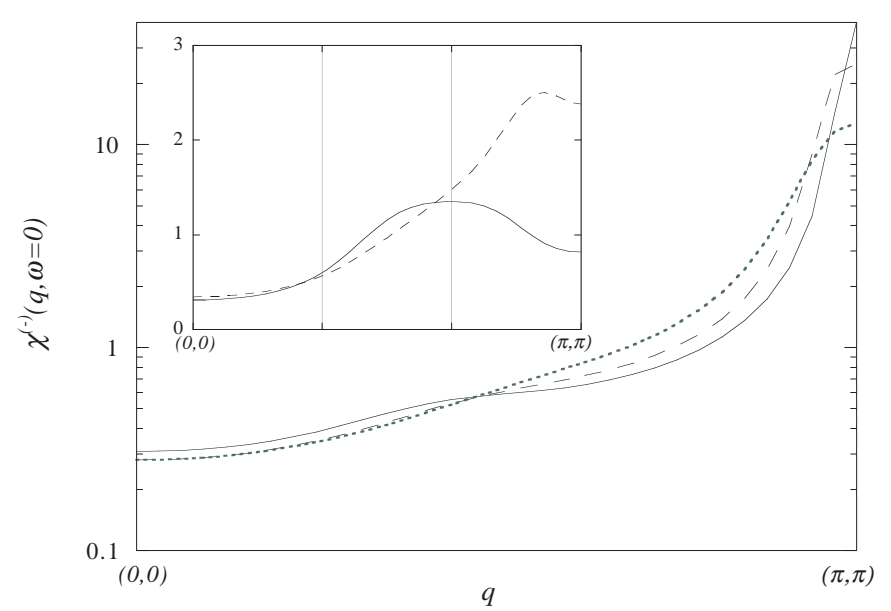

Fig. 5

Fig.3 (a) 